\title{
Un modèle en recherche clinique : la drépanocytose
}

Du malade à la protéine, de la protéine au gène, du gène aux tentatives thérapeutiques, la drépanocytose représente le modèle privilégié d'une recherche thérapeutique moderne.

Jean Rosa
Directeur de l'unité de génétique
moléculaire et d'hématologie de
l'Inserm (U.91).
Chef de service à l'hôpital
Henri-Mondor.

Jean Rosa

moléculaire et d'hématologie de l'Inserm (U.91).

Henri-Mondor.

\section{ADRESSE}

J. Rosa : Inserm U.91 et U.A. Cnrs 607, 558

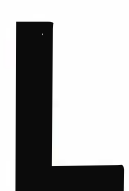

a drépanocytose ou anémie falciforme constitue actuellement un des modèles les plus avancés en matière de recherches diagnostiques, physiopathologiques et thérapeutiques. Les stratégies utilisées à son propos ont déjà été appliquées à l'étude de nombreuses autres maladies génétiquement déterminées et un grand nombre des enseignements tirés peuvent servir au développement de recherches dans les maladies acquises.

\section{Données de base}

La drépanocytose ou anémie falciforme [1] est une affection génétiquement déterminée à transmission autosomique récessive, caractérisée par la présence dans les globulès rouges d'une hémoglobine anormale : l'hémoglobine $\mathrm{S}$ (Hb S : $\left.\alpha_{2}{ }_{\beta}{ }_{2}\right)$, la mutation siégeant sur le gène codant pour la chaîne $\beta$, situé sur le chromosome 11. Les porteurs du trait sont, sauf circonstances exceptionnelles, bien portants. Plusieurs centaines de millions de sujets sont porteurs du trait dans les ethnies noires, très probablement en raison d'un avantage sélectif que ce trait procure vis-à-vis du paludisme. L'anémie falciforme survient chez les homozygotes $\mathrm{S} / \mathrm{S}$ et chez les doubles hétérozygotes, dont l'un des chromosomes porte la mutation $\$ 6$ Glu $\rightarrow$ Val caractéristique de l'Hb $S$ et l'autre, soit une mutation $\beta$ (ex. : Hb C, Hb D Los-Angeles), soit des altérations du type $ß$ thalassémique* ${ }^{*}$ La physiopathologie de l'affection est en apparence extrêmement simple (figure 1). L'Hb S, lorsqu'elle est désoxygénée, polymérise en longues fibres qui rigidifient et déforment le globule rouge en lui donnant une forme de faucille ("drepanos " en grec). Cette falciformation entraîne des troubles rhéologiques au niveau des capillaires à l'origine de multiples accidents thrombotiques qui émaillent la vie des drépanocytaires et finissent plus ou moins tôt par entraîner la mort. Cette maladie est généralement mal connue en France métropolitaine où vivent actuellement environ 50000 transmetteurs et environ 3000 homozygotes.

\section{Bases moléculaires de la falciformation}

L'hémoglobine A est une protéine extrêmement soluble présente à l'intérieur des globules rouges à la concentration considérable de $30 \%$. Au contraire, dans certaines conditions, la désoxyhémoglobine $\mathrm{S}$ se prend en un gel à l'intérieur duquel se forment de longues fibres d'hémoglobine s'associant en faisceaux qui déforment le globule rouge normalement biconcave. Le phénomène est réversible : lors de la réoxygénation, les fibres se dissocient et le globule oxygéné reprend forme et souplesse normales. Ce phéno-

\footnotetext{
- Le syndrome $\beta$ thalassémique est caractérisé par la synthèse insuffisante ou nulle de chaînes $\beta$ par le gène correspondant, quelle que soit la lésion moléculaire sous-jacente (mutation non-sens, délétion partielle ou totale, lésion d'un promoteur, entre autres).
} 


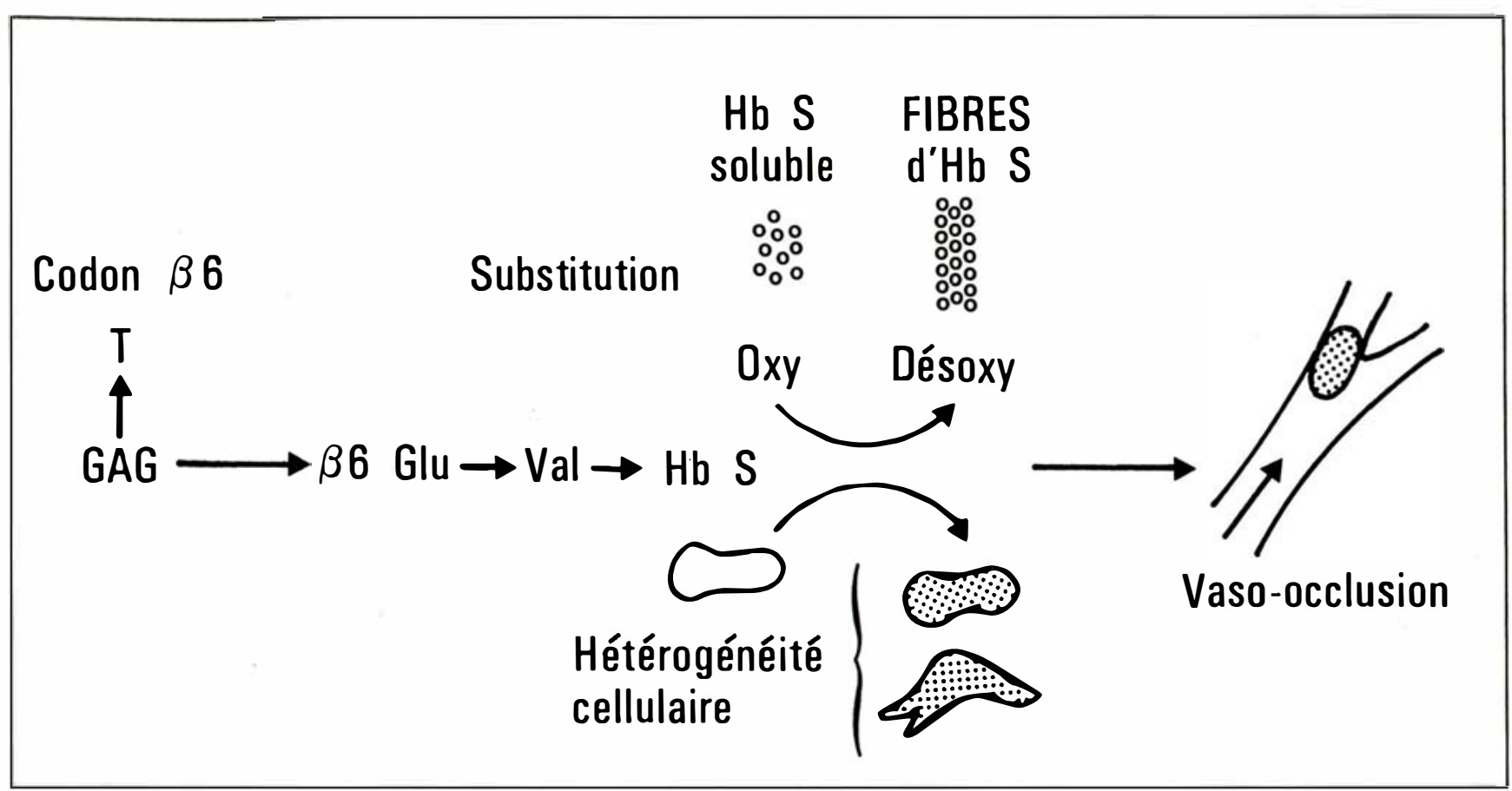

Figure 1. Dans le gène $\beta^{S}$, le nucléotide adénine du codon $n^{\circ} 6$ est remplacé par une thymine ce qui, au niveau protéique, amène la présence en position 6 d'une valine à la place de l'acide glutamique et donc I'Hb $S$. Alors que la mutation n'influence pas la solubilité de l'oxy $\mathrm{Hb}$ S, elle diminue dramatiquement celle de la désoxy-Hb S. Cette diminution de solubilité est liée à la formation de polymères insolubles faits d'agrégats de molécules de désoxy $\mathrm{Hb} S$. II en résulte par ailleurs une hétérogénéité cellulaire due au nombre différent de polymères suivant que les cellules sont jeunes ou vieilles. Les cellules contenant beaucoup de fibres peuvent s'accumuler dans un capillaire en formant un thrombus.

mène est d'autant plus remarquable que l'Hb $\mathrm{S}$ ne diffère que par un seul acide aminé de l'Hb $\mathrm{A}$, et qu'aucun des 450 autres mutants n'a un comportement identique, même ceux présentant une substitution Glu $\longrightarrow$ Val en un autre site que le $6^{\mathrm{e}}$ résidu ou portant sur le même résidu ß6 mais avec un acide glutamique remplacé par autre chose qu'une valine. L'étude de la structure, des propriétés et de la formation de ces polymères d'hémoglobine $S$ a fait l'objet d'un très grand nombre de travaux auxquels ont participé chimistes, cristallographes, physicochimistes, généticiens, etc. Les fibres sont visibles au microscope électronique qui montre de longs filaments réguliers. Edelstein a démontré [3] que chaque fibre était constituée par l'association de sept paires de filaments (figure 2) faits eux-mêmes de l'association de tétramères de désoxy-Hb $\mathrm{S}$.

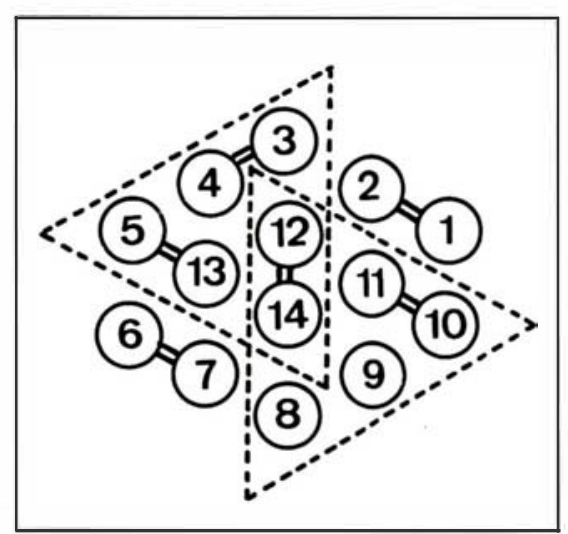

Figure 2. Section transversale d'une fibre d'Hb $S$ selon Edelstein. Chaque cercle représente une molécule présente dans un brin. II y a 14 brins dont 10 sont à l'extérieur. Les brins sont appariés 2 à 2 . Cette structure est extrêmement organisée et implique de très nombreux contacts intermoléculaires là l'intérieur d'une paire et d'une paire à l'autre). II y a aussi des contacts d'un étage à l'autre.
Les molécules ne sont pas orientées au hasard mais chaque molécule interagit très précisément avec une partie déterminée des molécules qui l'entourent, partie qui diffère suivant qu'il s'agit d'une molécule d'un même filament ou du filament homologue dans une paire, ou d'une molécule appartenant à un des autres brins (figure $3, p$. suivante). Si Ia valine $\beta 6$ de l'Hb $\mathrm{S}$ joue bien un rôle déterminant dans la formation de ce polymère, de très nombreux autres sites de l'hémoglobine sont également impliqués dans les contacts comme cela a été montré par spectres de diffractions de cristaux d'Hb $\mathrm{S}$.

Il est très difficile d'apprécier, parmi les centaines de contacts présents dans ces cristaux, ceux jouant un rôle dans le déterminisme de formation des fibres car, rappelons-le, la totalité de ces contacts sauf un (la valine 36 ) impli- 
que des résidus présents dans l'hémoglobine A qui, elle, ne se gélifie pas et ne polymérise pas. Pour préciser les contacts importants, cliniciens et biochimistes ont effectué un travail de bénédictins. Chaque double hétérozygotie a été étudiée d'un point de vue clinique et d'un point de vue biologique. De nombreuses hémoglobines, même si elles n'étaient pas associées in vivo à l'Hb $\mathrm{S}$, ont été testées in vitro mélangées avec celleci. Pour apprécier la formation des polymères, des méthodes de plus en plus sophistiquées ont été développées. Initialement, on a déterminé la concentration minimale à laquelle l'hémoglobine se gélifiait in vitro puis on a constaté que la prise du gel était précédée d'une phase de latence pendant laquelle s'effectuait un début de nucléation et la durée de cette phase s'est révélée être un paramètre important (delay time). Les polymères ayant une très faible affinité pour l'oxygène, les courbes de dissociation de l'hémoglobine sont déviées vers la droite, ce qui permet d'apprécier leur présence et de préciser leur temps d'apparition. Plus récemment, des méthodes beaucoup plus précises ont été développées, comme l'ultracentrifugation, qui isole les polymères d'un surnageant dont la concentration en hémoglobine est caractéristique de la tendance de l'hémoglobine à se gélifier. Enfin, on a pu détecter et quantifier les polymères intra-cellulaires par microspectrophotométrie précédée d'une désoxygénation des cellules par photolyse au laser, ou encore en analysant par RMN au carbone-13 les contenus cellulaires. L'équipe de Nagel initia l'étude des contacts décelés par l'analyse des mutants $\beta$, tandis que les Benesch étudièrent les contacts dans le polymère à l'aide de mutants $\alpha$. Pour notre part, ayant identifié un nombre important de mutants nous les avons testés in vitro avec l'hémoglobine $\mathrm{S}$. De nombreux mutants se sont révélés neutres vis-à-vis de la formation du polymère, telle l'hémoglobine Siriraj ß7 Glu $\rightarrow$ Val [4],
Figure 3. Organisation des doubles brins dans les cristaux d'hémoglobine $\mathbf{S}$ (obtenus in vitro) à gauche, ot dans les fibres des drépanocytes (à droito). Chaque cercle représente une molécule d'hémoglobine. Les deux structures sont très comparables, à la différence près que les brins s'enroulent en spirale dans la fibre alors qu'ils sont paralleles dans le cristal. Le pointillé représente l'axe de la fibre légèrement incliné par rapport à l'axe vertical des brins du cristal. Dans les deux cas, il s'agit d'une organisation extrêmement élaborée, chaque molécule étant en contact précis avec les molécules l'entourant: contacts dits axiaux pour les molécules superposées dans un même brin lessentiellement des contacts entre chaînes $\alpha$ et $B)$, contacts dits latéraux entre molécules d'un brin à l'autre. C'est là qu'une seule des 2 valines B6 d'un tétramère donné est impliquée; telle la partie mâle d'un bouton pression, elle vient se fixer dans une cavité présente sur une chaîne B de la molécule d'Hb S en face d'elle dans l'autre brin, au niveau de la leucine B85. Chaque molécule est "donneuse " et "receveuse ". Sur cette figure, la molécule (a) représentée en gris clair enfonce sa valine $B 6$ dans la molécule (b) hachurée tandis que celle-ci enfonce sa valine $\beta 6$ dans le site $\beta 85$ de la molécule (c), en gris foncé. Ces contacts sont détaillés sur la figure 4.

située à proximité immédiate du résidu muté dans l'Hb $\mathrm{S}$ et présente le même type de substitution Glu $\rightarrow$ Val.

Ce résultat in vitro corrélait parfaitement avec l'étude clinique, le double mutant S/Siriraj détecté à la Martinique ayant une excellente santé. De même, en étudiant les hémoglobines "Le Lamentin " $(\alpha 20)[5]$ et "Stanleyville " $(\alpha 78)$ [6], nous avons montré que ces résidus qui participent

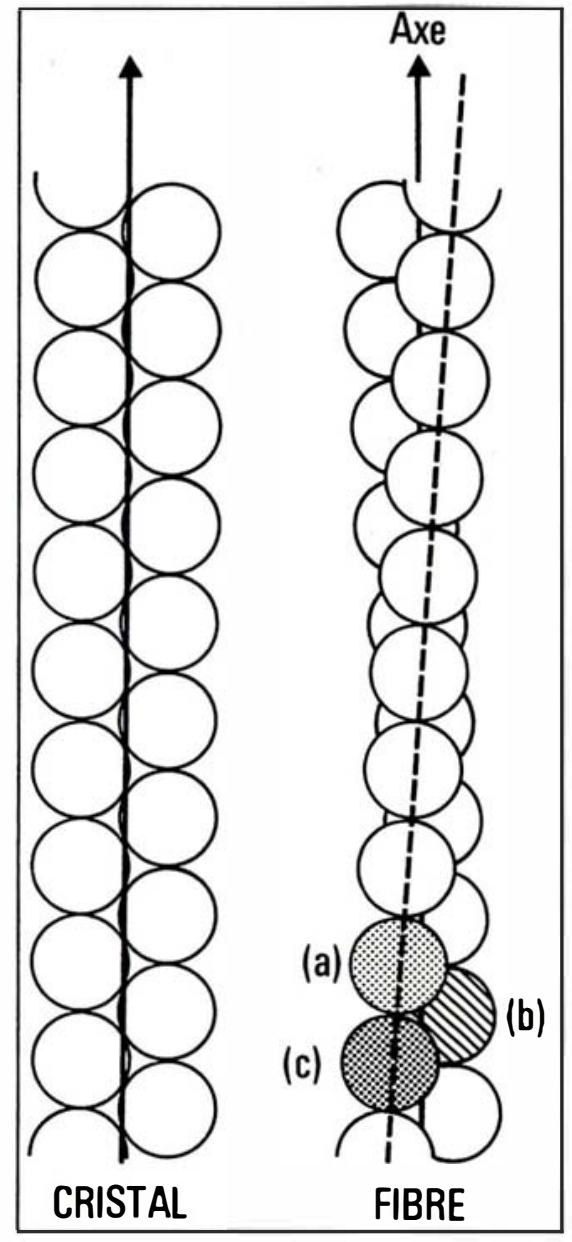

aux contacts intermoléculaires dans le cristal d'hémoglobine S sont bien impliqués dans les contacts axiaux de la fibre d'hémoglobine S. Dans le cristal, l'histidine $\alpha 20$ interagit avec l'acide glutamique $ß 22$ de la molécule sous-jacente ; la glutamine $\alpha 20$, par contre, présente dans l'hémoglobine Le Lamentin, ne peut pas échanger de liaison ionique de ce type et la cohésion du polymère est diminuée. C'est exactement ce 
Figure 4. Carte en coordonnées polaires représentant l'hémisphère d'une molécule d'hémoglobine orientée vers l'intérieur des doubles brins. Les régions représentées en gris clair et gris foncé indiquent les zones de contact avec les molécules marquées des mêmes symboles en bas de la figure 3. A l'intérieur de ces zones de contact, les chiffres dans les cercles localisent des résidus, certains correspondant à des mutations dont le nom est inscrit dans un rectangle en trait plein (ex. : Toulouse) quand la mutation modifie la formation des fibres et en pointillé (ex. : S-Travis) quand elle est indifférente. On voit clairement que le site donneur est situé sur la chaîne $\beta_{2}$, alors que le site receveur est situé sur la chaîne $\beta_{1}$ dite en "r trans ". Les chiffres dans les losanges répertorient les contacts entre fibres représentées sur la figure 2.

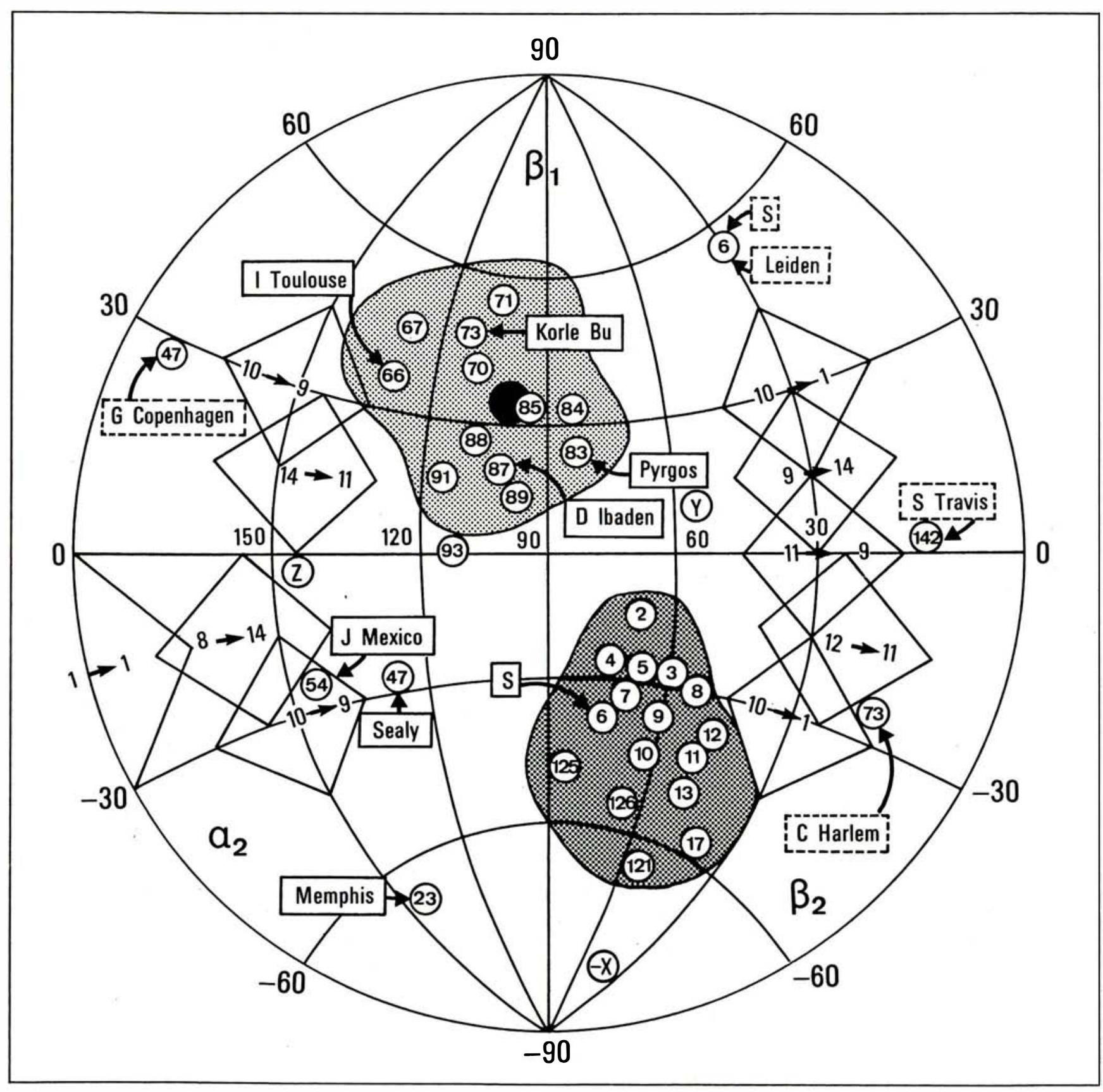




\section{RÉFÉRENCES}

1. Sergeant GR. Sickle Cell Disease. London : Oxford Press, 1985.

2. Bunn HF, Forget BG. Hemoglobin: Mole cular, Genetic and Clinical Aspects. Philadelphia : W.B. Saunders, 1986.

3. Edelstein JS. Les aspects moléculaires de la drépanocytose. La Vie des Sciences $1984 ; \mathrm{n}^{\circ} 2$ : 89-96.

4. Rhoda MD, Arous N, Garel MC, et al. Interaction of hemoglobin Siriraj with hemoglobin S : a mild sickle cell syndrome. Hemoglobin $1986 ; 10$ : 21-31.

5. Rhoda MD, Blouquit Y, Caburi-Martin J, et al. Effects of the $\alpha 20$ mutation on the polymerization of Hb S. Biochim Biophys Acta 1984 ; $786: 62-6$

6. Rhoda MD, Martin J, Blouquit Y, Garel MC, Edelstein SJ, Rosa J. Sickle cell hemoglobin fiber formation strongly inhibited by the Stanleyville II mutation ( $\alpha 78$ Asn $\rightarrow$ Lys). Biochem Biophys Res Commun 1983 ; 111 : 8-13.

7. Montplaisir N, Merault G, Poyart C et al. Hb S-Antilles $\left(\alpha_{2} \beta_{2} 6 \mathrm{Glu} \rightarrow\right.$ Val $23 \mathrm{Val} \rightarrow$ Ileu) : A new variant with lower solubility than $\mathrm{Hb} \mathrm{S}$ and producing sickle cell disease in heterozygotes. Proc Natl Acad Sci USA 1986 (In press)

8. Godeau JF, Beuzard Y, Cacheleux J, Brizard CP, Gibaud A, Rosa J. Association of hemoglobin Saint Etienne $\left(\alpha_{2} \beta_{2} 92\right.$ F8 His $\rightarrow$ GIn) with hemoglobins $A$ and F. J Biol Chem 1976 ; 61 : 263-9.

9. Rosa J, Beuzard Y, Hercules J. Development of therapeutic agents for sickle cell disease. INSERM Symposium. Amsterdam : North Holland, 1979.

10. Beuzard Y, Charache S, Galacteros F. Approaches to the therapy of sickle cell anaemia. Éditions des Colloques INSERM, 1986.

11. Poyart C, Bursaux E, Teisseire B, Freminet A, Duvelleroy M, Rosa J. Hemoglobin Creteil : oxygen transport by erythrocytes in vitro and in vivo studies in a high oxygen affinity mutant hemoglobin. Ann Intem Med 1978 ; 88 : 758-63.

12. Hassan W, Beuzard Y, Rosa J. Inhibition of erythrocyte sickling by cystamine, a thiol reagent. Proc Natl Acad Sci USA $1976 ; 73$ :

que les mesures du mélange S/Le Lamentin nous ont indiqué. De la même manière, le remplacement de l'asparagine $\alpha 78$ par une lysine dans l'Hb Stanleyville supprime une interaction hydrophobe et diminue la tendance à la gélification.

Des renseignements également très importants ont pu être obtenus par l'étude de molécules doublement mutées, c'est-à-dire portant en plus de la mutation $S$ une deuxième modification sur la même chaîne $ß$. Contrairement à l'Hb C-Harlem qui polymérise moins que l'Hb $\mathrm{S}$, l'Hb C-Ziguinchor que nous avons isolée a strictement le même comportement que l'Hb $\mathrm{S}$ malgré l'addition en $B 58$ de la substitution glumatique $\rightarrow$ valine. Plus récemment, nous venons de déceler, à la Martinique, une super hémoglobine $\mathrm{S}$ : l'hémoglobine SAntilles $\alpha_{2} ß_{2} 6 \mathrm{Glu} \rightarrow \mathrm{Val} ; 23$ Val $\rightarrow$ Ileu [7], où le remplacement d'une valine de l'hélice $B$ augmente la polymérisation. L'effet aggravant est tellement important que la désoxyhémoglobine S-Antilles polymérise en présence d'hémoglobine $A$ et que les hétérozygotes $\mathrm{A} / \mathrm{S}$ Antilles présentent un syndrome drépanocytaire contrairement aux hétérozygotes A/S. L'Hb SAntilles ne pouvant être distinguée de l'Hb S par les méthodes électrophorétiques utilisées ordinairement, il est possible qu'elle soit à l'origine de ces cas de "soidisant " hétérozygotes $\mathrm{A} / \mathrm{S}$ malades, qui ont soulevé d'intenses polémiques tant cliniques que médico-légales. La figure 4, page précédente, représente la carte polaire des contacts de l'Hb S établie par Edelstein. On voit que le site 6 de la mutation $\mathrm{S}$, site donneur, s'emboîte dans un site receveur centré par le résidu 85 mais où participe le résidu 73 modifié dans l'hémoglobine Korlebu et dans l'hémoglobine C-Harlem. Il est tout à fait remarquable que tous les contacts axiaux soient communs à l'hémoglobine $\mathrm{A}$ et à l'hémoglobine $\mathrm{S}$, ce qui implique que la fixation latérale d'une première molécule d'hémoglobine doit précéder la fixation des molé- cules dans le plan axial. Il est clair que de telles cartes sont d'un intérêt capital pour le développement rationnel de drogues antifalciformantes et que, si on arrivait par exemple à intercaler un corps chimique au niveau du résidu 85 , on empêcherait le résidu Val 6 d'une autre molécule de venir s'y fixer. Nous verrons les difficultés que ce genre d'exercice entraîne.

\section{Bases cellulaires de la drépanocytose}

Lésion génétique ponctuelle, protéine substituée dont la configuration désoxy (forme $\mathrm{T}$ ) polymérise, drépanocyte, globule rouge monstrueux déformé, rigide, aux propriétés rhéologiques profondément perturbées, telles sont les caractéristiques de la drépanocytose (figure 1) [2]. La structure, la cinétique de formation et les propriétés des drépanocytes ont fait l'objet d'études nombreuses. Les résultats observés et les techniques développées à propos de ces études ont d'ailleurs eu des retombées sur des maladies autres que la drépanocytose, telles que le diabète ou l'athérosclérose.

La population érythrocytaire des homozygotes drépanocytaires est extrêmement hétérogène, chez un même sujet et d'un sujet à l'autre. Cette hétérogénéité est très bien objectivée par les profils de gradients de densité (figure 5) qui séparent un nombre de couches très supérieur à celui obtenu avec des sangs témoins. Ce fait est dû à la présence de nombreux réticulocytes et de cellules plus denses que des cellules normales. Elles présentent une déformation permanente (irreversible sickle cells), sont très déshydratées, riches en calcium, pauvres en potassium. Leurs membranes sont intactes et, rehydratées, elles reprennent un aspect biconcave, les plus âgées ayant des altérations du cytosquelette. Elles se forment probablement à la suite de cycles répétés de falciformation et de retour à la normale. Au cours de la désoxygénation dans les capillaires périphériques, la polymérisation ne fait que s'initier ou plus précisé- 
Figure 5. Séparation par centrifugation sur Stractan de globules rouges d'un homozygote $\mathbf{S} / \mathbf{S}$. La densité des cellules augmente de haut en bas. La couche inférieure contient les "irreversible sickle cells" visibles en bas et à droite et est à comparer avec les cellules jeunes (en haut et à droite). Cette couche n'existe pas dans des séparations de sang de sujets AA. Chez ces derniers, les deux couches principales centrales n'en forment qu'une seule.

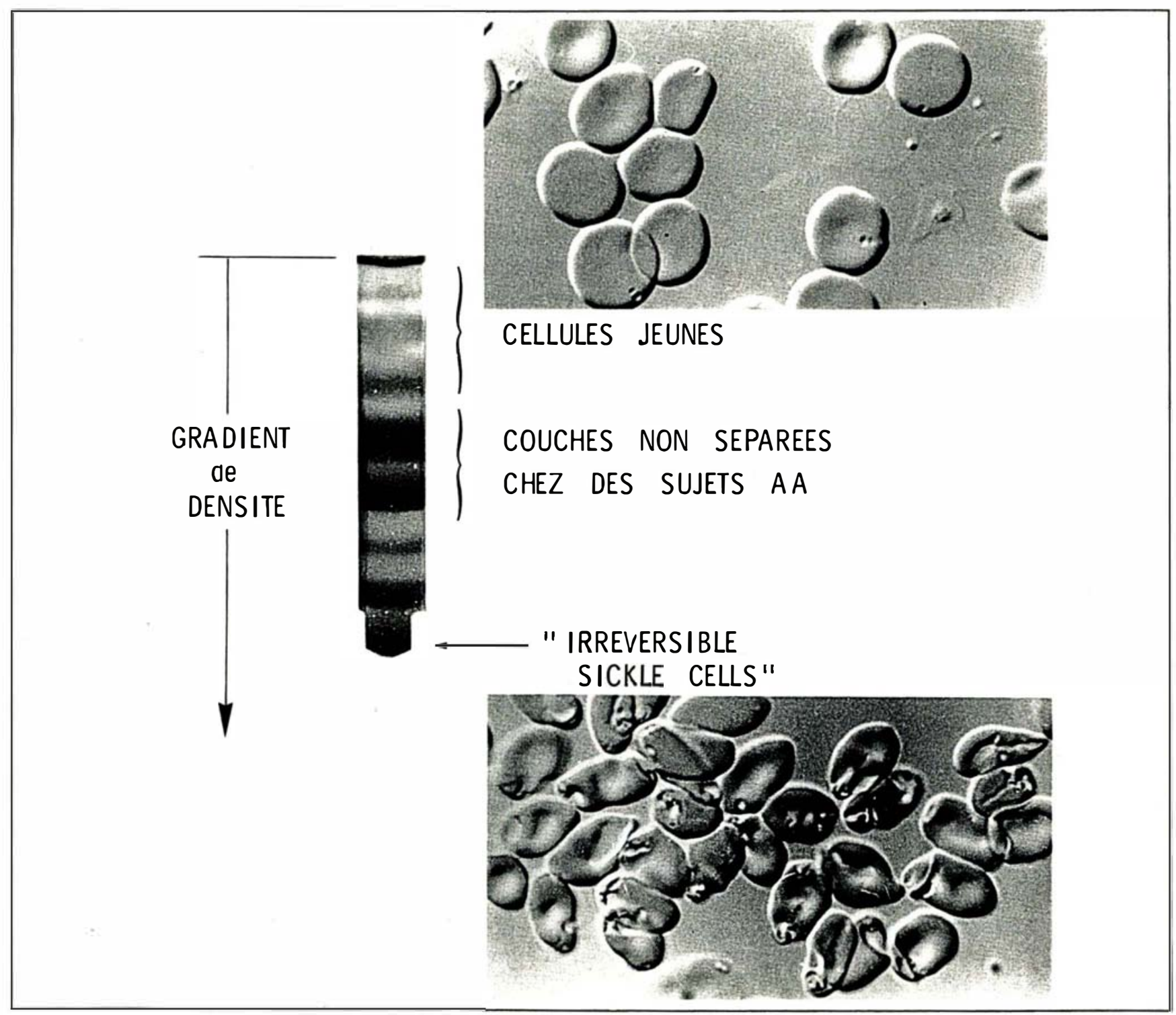


ment se poursuivre (car il y a déjà des polymères à des pressions élevées d'oxygène) et ne se complète qu'après le retour des hématies dans le sang artériel. Au cours de la polymérisation, le globule se déshydrate partiellement, avec une fuite de potassium incomplètement équilibrée par l'entrée du sodium et une captation irréversible de calcium qui se fixe dans un compartiment indépendant. Ce phénomène n'est expliqué ni par un dysfonctionnement de l'ATPase calcium-dépendante, ni par un déficit énergétique dans le drépanocyte. La cinétique de falciformation est influencée par un très grand nombre de paramètres.

La nature des hémoglobines éventuellement présentes en association avec l'hémoglobine $\mathrm{S}$ peut intervenir, comme nous l'avons vu plus haut pour l'Hb A et d'autres hémoglobines anormales. L'hémoglobine fotale (HbF) est un très puissant inhibiteur de la polymérisation, ce qui, ajouté au fait que la quantité d'Hb S est plus faible que chez l'adulte, contribue à l'absence de troubles chez le fœtus et le nouveau-né. C'est aussi la situation rencontrée chez certains sujets présentant la prédisposition génétique dite " persistance héréditaire d'hémoglobine fœtale $(\mathrm{PH}$ HF) hétérocellulaire ". Les hétérozygotes pour cette disposition ont un taux légèrement plus élevé d'hémoglobine F et de " $F$-cells" que les sujets normaux. Par contre, en cas d'hémolyse, comme nous avons pu le démontrer en particulier au cours d'une anémie hémolytique due à une hémoglobine instable, l'Hb St-Étienne [8], la synthèse d'hémoglobine fœtale peut très notablement s'élever $(\mathrm{Hb}$ $\mathrm{F}>15 \%$ ). La PH HF hétérocellulaire est fréquente chez les Noirs et constitue certainement un facteur de protection contre la drépanocytose, atténué cependant car la répartition d'hémoglobine fotale y est hétérogène, laissant certains globules avec des taux bas d'Hb F. Il n'en demeure pas moins que ce modèle est particulièrement stimulant comme voie d'approche de thérapeutiques génétiques et plusieurs groupes cherchent à locali- ser le/les locus génétique(s) responsable(s) de ce phénomène.

- L'abaissement du pH déplaçant la courbe de dissociation vers la droite augmente pour une $\mathrm{pO}_{2}$ donnée le pourcentage de désoxy $\mathrm{Hb} \mathrm{S}$ et favorise donc polymérisation et falciformation. Cet effet joue très vraisemblablement un rôle déclenchant en cas d'acidose chez l'enfant drépanocytaire.

- La concentration moyenne en hémoglobine est également un paramètre important dans le déclenchement de la falciformation. Des études cinétiques très fines ont montré la très grande dépendance de la polymérisation vis-à-vis de la dilution intracellulaire. Tout ce qui abaisse la concentration globulaire en hémoglobine diminue les risques de falciformation, d'où la protection paradoxale que confèrent les anémies associées à la drépanocytose, telles les anémies ferriprives et surtout les anémies hypochromes dues aux $\alpha$ thalassémies* particulièrement fréquentes dans les ethnies noires (nous avons ainsi trouvé $22 \%$ d' $\alpha_{2}$-thal et $2 \%$ d' $\alpha_{1}$-thal en Martinique et en Guadeloupe à la suite de dépistage néonatal systématique). D'autres paramètres peuvent encore jouer un rôle, par exemple des anomalies de la microcirculation augmentant le shunt pulmonaire.

- Enfin, l'adhésivité entre les drépanocytes et les cellules de l'endothélium vasculaire est augmentée, ce qui pourrait intervenir dans le déclenchement des crises drépanocytaires. Un tel phénomène a servi de modèle à l'étude de l'adhésivité anormale aux cellules endothéliales des globules rouges de malades diabétiques ayant un taux d'hémoglobine glycosylée très élevé.

\section{Recherches thérapeutiques}

Les paragraphes précédents ont montré la complexité des bases physiopathologiques de la drépa-

\footnotetext{
- Syndromes généliquament délerminés caractérisés par un déficit en synthèse de chaine $\alpha$. Les formes présentes dans l'ethnie noire sont les $\alpha_{2}$ thal : $\alpha /-, \alpha / \alpha$ et les $\alpha_{1}$ thal : $\alpha /$ - $\alpha$-.
}

18. Weatherall D. Prenatal diagnosis of inherited blood diseases. In : Clinics in Hematology. 
nocytose. Certaines ont servi de point de départ à des recherches thérapeutiques. Malgré l'étendue des progrès réalisés dans l'application des techniques les plus récentes de "drug design "*, de greffes de moelle et de génie génétique, le traitement de la drépanocytose reste essentiellement à l'heure actuelle symptomatique ou préventif, ce qui ne signifie pas qu'il n'y ait pas eu de très grandes améliorations quant à sa morbidité.

(a) Les recherches médicamenteuses [9, 10]. Hormis les essais initiaux de vaso-dilatateurs ne reposant pas sur des bases expérimentales très poussées, les essais de développement de drogues ont été orientés dans trois directions : l'augmentation de l'affinité pour l'oxygène des globules, l'inhibition des contacts intermoléculaires et la diminution de la concentration hémoglobinique intracellulaire.

En augmentant l'affinité pour l'oxygène de l'hémoglobine, on vise à augmenter le nombre de molécules en conformation " oxy R ", conformation qui ne se prête pas à la polymérisation. L'idée était séduisante au départ et de très nombreuses drogues ont été développées dans ce sens, développement favorisé par l'étude de très nombreux mutants à affinité augmentée, tels que les hémoglobines Créteil, Hôtel-Dieu, ou Pitié-la-Salpêtrière [11]. Ces études avaient permis de définir une série de mécanismes déviant vers la gauche l'équilibre $\mathrm{R} \leftrightarrow$ $\mathrm{T}^{* *}$, que ce soit en inhibant la fixation de 2,3 DPG** dans la cavité centrale, en favorisant l'entrée de l'oxygène dans la poche

\footnotetext{
" "Drug design ": méthode de conception des drogues en fonction de la stéréochimie de leurs cibles, faisant largement appel à l'informatisation.

** 2,3 DPG = 2,3, diphosphoglycérate, métabolite de la glycolyse dont la concentration est la plus élevée dans les globules rouges. Cet agent se lie à l'hémoglobine, dont il diminue l'affinité pour l'oxygène, facilitant ainsi sa libération dans les tissus. La configuration $R$ et $T$ de l'hémoglobine définissant, selon le modele de Monod et Changeux, les états allostériques à haute at à basse affinité pour l'oxygène, on peut dire que le 2,3 DPG déplace l'équilibre $R \longrightarrow T$ vers la droite (forme T), son absence déplaçant au contraire l'équilibre vers la gauche (forme $R$ ).

$\mathrm{m} / \mathrm{s} \mathrm{n}^{\circ} 10$ vol. 2, décembre 86
}

de l'hème ou en bloquant " l'articulation $\alpha_{1} \beta_{2}$ " au niveau de laquelle se produisent les mouvements permettant de passer de la configuration " $\mathrm{R}$ " (relachée, oxy) à la configuration contrainte " $T$ " (désoxy). Le cyanate, première drogue essayée, ainsi que de nombreuses autres y compris l'aspirine, peuvent, au moins in vitro, atteindre cet objectif. En fait, on s'est heurté aux exigences très complexes de ce type d'approche. Il faut en effet que la drogue soit absorbable par voie digestive, non toxique, non active sur d'autres cellules que le globule rouge, qu'elle passe aisément la membrane érythrocytaire, qu'elle ne dénature pas l'hémoglobine, exigences en effet particulièrement complexes du fait de la concentration élevée de l'hémoglobine et de son énorme masse totale. Yves Beuzard et ses collaborateurs ont développé dans notre groupe une approche originale pour tenter de résoudre ce problème en jouant sur la présence d'un seul groupe $\mathrm{SH}$ (sulfhydrile) réactif par tétramère d'Hb, situé de plus dans une région directement impliquée au cours de la transition $\mathrm{T} \leftrightarrow \mathrm{R}$. Partant de la cystamine, dont nous avions montré avec W. Hassan qu'elle inhibait la falciformation et augmentait la concentration minimale de gélification [12], ils ont en collaboration avec les organiciens de Sanofi testé un très grand nombre de disulfures. Plusieurs se sont révélés être de remarquables inhibiteurs de la polymérisation mais aucun jusqu'ici n'a rempli toutes les conditions pré-requises citées plus haut, en raison notamment de leur fixation sur d'autres $\mathrm{SH}$ que ceux de l'hémoglobine [13]. Par ailleurs, sur le plan théorique, il n'est pas sans effet pervers d'augmenter l'affinité pour l'oxygène de globules drépanocytaires car on risque de déclencher, comme chez les porteurs d'hémoglobines à affinité augmentée, une polyglobulie réactionnelle [2] qui, en augmentant la viscosité sanguine des patients, peut favoriser l'apparition d'accidents vaso-occlusifs.

Il est également extrêmement séduisant d'essayer de développer des drogues inhibant les contacts du polymère et nous avons vu à propos des mutants combien la substitution d'un seul acide aminé au niveau d'un contact pouvait modifier la formation et la stabilité du polymère. Mais il est actuellement hors de portée de pouvoir induire ce type de modification in vivo. Nous avons vu combien la cinétique de formation du polymère était corrélée à la concentration intracellulaire en hémoglobine et l'effet bienfaisant des facteurs favorisant une baisse de la concentration moyenne en hémoglobine $(\mathrm{CMH})$ tels que l'anémie ferriprive et surtout les $\alpha$-thalassémies. De là est née l'idée d'essayer d'obtenir artificiellement une baisse de la $\mathrm{CMH}$ chez les homozygotes SS, soit en abaissant l'osmolalité plasmatique, soit en augmentant la perméabilité de la membrane érythrocytaire. Un certain nombre de drogues ont été essayées dans ce sens mais, ici encore, les résultats satisfaisants obtenus in vitro n'ont jusqu'à maintenant pas été suivis de résultats in vivo probants [10]. Le traitement extra-corporel des globules de patients a cependant permis d'obtenir quelques résultats en utilisant de très fortes concentrations de drogues (toxiques in vivo). Ces essais restent encore du domaine de l'expérimentation thérapeutique.

(b) La greffe de moelle. Surtout essayée dans les ß-thalassémies homozygotes, elle peut être appliquée au traitement de la drépanocytose [14]. La très haute technologie nécessaire, son coût, la survenue de complications graves, en font un traitement inapplicable dans les pays en voie de développement et finalement peu utilisé dans les pays à économie forte. (c) La modification de l'expression du génome hémoglobinique. Des recherches dans ce sens ont été entreprises. Nous avons vu que l'Hb $\mathrm{F}$ était un très puissant inhibiteur de la falciformation. Ses nombreuses substitutions en acides aminés comparé à l'Hb $\mathrm{S}$, font qu'elle ne se prête pas à une incorporation dans le gel dont elle stoppe, au-delà de $20 \%$, la formation. Les résultats cliniques 
confirment ces données et les sujets $\mathrm{S} / \mathrm{S}$ présentant une persistance héréditaire d'hémoglobine fœtale associée, ont une protection évidente. Différentes drogues utilisées dans le traitement de leucémies telles que la 5-aza-cytidine et l'hydroxyurée étaient connues pour augmenter chez les sujets traités le taux d'hémoglobine fœtale. Après des essais sur le singe, elles ont été essayées chez des drépanocytaires [15, 16]. Les résultats furent intéressants mais non concluants : l'élévation observée d'hémoglobine fotale a été variable d'un sujet à un autre mais le plus souvent faible et le traitement n'a pas semblé diminuer de façon manifeste le nombre des crises. Le mécanisme d'action n'est d'ailleurs pas clairement défini : mise en activité de gènes $\gamma$ de l'hémoglobine fœtale par déméthylation ou action sur le cycle cellulaire amenant la moelle à libérer des cellules relativement immatures encore partiellement programmées pour la production d'hémoglobine fœetale ? (d) La greffe de gènes [17]. Estelle envisageable comme traitement futur de la drépanocytose? Les problèmes posés sont com- muns à toutes les greffes de gènes qui peuvent être envisagées ; ce sont ceux de l'innocuité du vecteur et de l'insertion proprement dite et celui de l'obtention d'une expression suffisante. On sait que, sur le plan expérimental, certains résultats ont déjà été obtenus, mais le traitement génétique d'une maladie comme la drépanocytose pose un certain nombre de problèmes particuliers. Il ne s'agit pas ici en effet de suppléer la carence de production d'un gène comme pour la maladie de Lesch-Nyan*. Dans le cas de la drépanocytose, le produit du gène anormal $\beta S$ est directement à l'origine des accidents. La réussite de la greffe du gène $B A$ normal ne suffirait pas à supprimer ces accidents sauf si les deux gènes (le gène $\beta^{S}$ et le gène $\beta^{A}$ introduit) étaient exprimés simultanément et dans les mêmes cellules, comme c'est le cas chez les hétérozygotes A/S " naturels". On voit mal dans l'état actuel des connaissances comment y parvenir. Une autre difficulté considérable tient au fait que, non seulement le gène introduit doit pouvoir obéir à une régulation, mais encore que son expression doit être exacte- ment coordonnée avec celle des gènes $\alpha$, sous peine de créer un déséquilibre entre le taux des chaînes $\alpha$ et $\beta$ qui entraînerait artificiellement un syndrome thalassémique. Quels que soient les progrès des recherches actuelles en matière de promoteurs et de séquences stimulatrices des gènes eucaryotes, quel que soit l'immense intérêt que suscite l'élucidation des phénomènes cis et trans de contrôle de l'expression des gènes, il est peu probable que l'on maîtrise le type de greffes de gènes nécessaire au traitement de maladies telles que la drépanocytose dans les années à venir. Si l'on voulait se risquer à faire des paris, il serait probablement moins aléatoire de miser sur les modifications par génie génétique du contrôle des systèmes de régulation de l'expression des gènes $\gamma$ fœtaux. Les recherches coordonnées partant de l'étude du matériel naturel constitué par les très nombreux mutants thalassémiques ou atteints de persistance

- Maladie de Lesch-Nyan : déficil congénital en hypoxanthineguarylphophoribosyltransférase (HGPRT). Cette maladie, liée au chromosome $X$, entraine une goutte sévère, associée à d'autres symplômes systémiques.

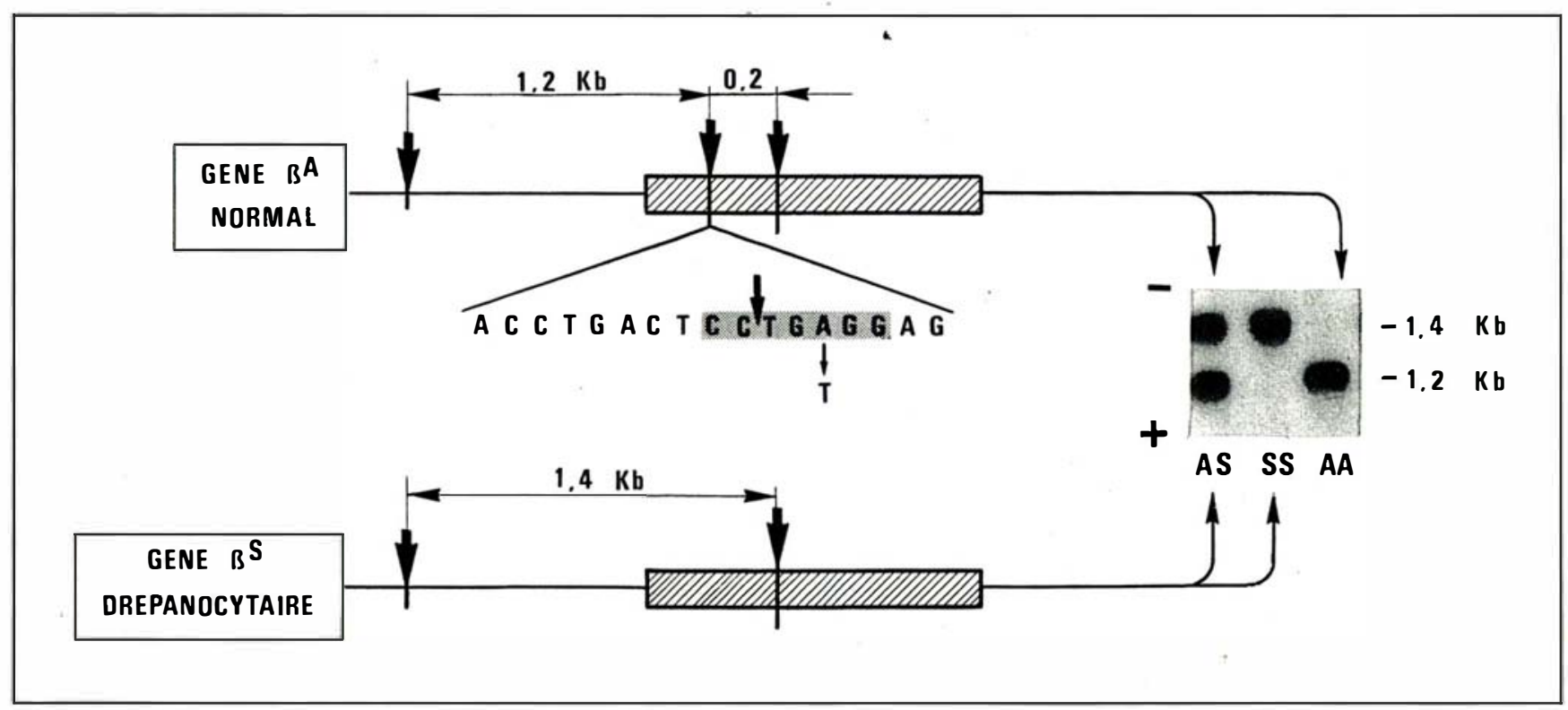

Figure 6. Diagnostic prénatal de la drépanocytose par génie génétique. Les enzymes Mst II ou CVN I sont utilisées pour la détermination de l'existence de la mutation $B S$. Lorsque celle-ci est présente, elle modifie la séquence CCT GA GG, site de coupure pour l'enzyme et supprime la coupure à ce niveau. Le fragment de restriction est donc allongé et ce changement de longueur peut être détecté par "Southern blot " à l'aide d'une sonde radioactive d'ADNc. Dans le cas présent c'est l'enzyme CVN I qui a été utilisée. 
héréditaire d'hémoglobine fotale, la détermination des séquences de régions régulatrices, les recherches en cours sur les molécules agissant en trans, peuvent laisser espérer avoir, dans un avenir raisonnable, des substances médiatrices capables d'induire à la demande la synthèse d'hémoglobine fotale. Ce faisant, les perspectives de traitement de la drépanocytose, mais aussi des thalassémies, seraient radicalement modifiées... et probablement aussi celles d'autres maladies monogéniques à systèmes de gènes redondants.

\section{Prévention}

Le diagnostic prénatal de la drépanocytose $a$, en quelques années, progressé de façon foudroyante [18]. Réalisé vers les années 76 par radio-chromatographie des hémoglobines sur sang de cordon de fœtus " à risques ", prélevé à la $20^{e}$ semaine par fotoscopie, il est pratiqué maintenant sur l'ADN de trophoblastes prélevés à la $66^{\mathrm{e}}-8^{\mathrm{e}}$ semaine par simple aspiration. Le diagnostic est assuré par la différence de longueur des fragments de gènes $\beta^{A}$ et $\beta S$ clivés par les enzymes de restriction CVN I ou MST II : $1,2 \mathrm{~kb}$ pour le gène $\beta A ; 1,4 \mathrm{~kb}$ pour le gène $B S$ (figure 6 ) par suite de la disparition de la sensibilité à l'enzyme due à la transition $\mathrm{A} \rightarrow$ $\mathrm{T}$ de la mutation drépanocytaire. Il s'agit d'un diagnostic direct (il n'est pas nécessaire d'analyser l'ADN des parents). La méthode, parfaitement maîtrisée à l'heure actuelle (nous avons effectué ainsi plus de 150 diagnostics dans le laboratoire de biochimie de l'hôpital Henri-Mondor), a permis de très bien roder ce type de méthodologie pour diagnostic prénatal des maladies génétiques. Cependant, la drépanocytose due à une mutation unique constitue une maladie génétique exceptionnelle. Pour transposer aux autres maladies monogéniques justifiant un diagnostic prénatal, telles que les hémophilies ou les dystrophies musculaires, il faut se référer non pas à la drépanocytose en particulier mais au modèle plus général " hémoglobinopathies" dont on $\mathrm{m} / \mathrm{s} n^{\circ} 10$ vol. 2 , décembre 86 connaît plus de 450 lésions moléculaires différentes. Ceci rend impossible tout diagnostic direct et impose la méthode infiniment plus contraignante de l'analyse par polymorphismes de restriction*.

\section{Conclusion}

La recherche clinique est souvent l'objet d'âpres controverses portant sur le concept lui-même et sur une soi-disant déshérence. On tombe néanmoins d'accord pour définir comme telle une recherche partant du malade et lui revenant. Les recherches sur la drépanocytose sont à notre avis exemplaires en ce sens que les recherches, tant cellulaires que moléculaires, ont toujours été précédées d'une étude très poussée des manifestations cliniques.

Aucune maladie n'a bénéficié d'un ensemble d'études aussi cohérentes, moléculaires, cellulaires, génétiques, cliniques, diagnostiques et thérapeutiques, que la drépanocytose. Les résultats obtenus sont considérables. Une conclusion s'en dégage. L'homme et l'homme malade constituent un système d'une extrême complexité. Une lésion génétique simple entraînant l'apparition d'une anomalie protéique parfaitement définie touchant la protéine la plus étudiée, s'est révélée entraîner des perturbations extraordinairement complexes à l'échelle de l'individu entier. Des interactions génétiques ou non génétiques multiples se produisent, rendant la maladie extrêmement polymorphe et la survenue des accidents aléatoire. Aucun autre système ne se prêtait mieux à un développement logique de thérapeutique ( $d r u g$ design). Malgré un nombre considérable de résultats, la solution n'est toujours pas trouvée. Il est très vraisemblable que cette "Saga " pourra inspirer de nombreuses démarches à propos d'autres affections, génétiquement déterminées ou non. L'histoire que nous venons de décrire laisse à penser que cela nécessitera encore beaucoup d'efforts

- Voir lexique m/s $n^{\circ} 3$, vol. 2, p. 152.

\section{Summary}

The present report summarizes the most recent data on the structure and the formation of fibers of deoxy hemoglobin S. It is shown, using some examples, how various abnormal hemoglobins can be used to identify the most important contacts in the hemoglobin S polymer formation. The cellular events producing the irreversible sickle cells are reviewed. Antisickling drug design is explained using some examples. Different therapeutic approaches using molecular biology are discussed.
Remerciements. Ce travail n'a été possible que grâce aux supports de l'INSERM, du CNRS, du ministère de la Recherche, de l'Université Paris Val-de-Marne, de la Fondation pour la Recherche Médicale et naturellement à la participation des nombreux chercheurs et techniciens de l'unité U.91 et de son annexe aux Antilles.

\section{TIRÉS A PART}

J. Rosa : Inserm U.91 et U.A. Cnrs 607, hôpital Henri-Mondor, 94010 Créteil. 